\title{
A state of the art on the most relevant patents in vertical transportation in buildings
}

\author{
Pablo Cortés ${ }^{1 *}$, José Guadix, Jesús Muñuzuri \\ ${ }^{1}$ Ingeniería Organización. Escuela Superior Ingenieros. University of Seville \\ c/ Camino de los Descubrimientos s/n. \\ E-41092. Seville - SPAIN \\ *Corresponding author: \\ Email: pca@esi.us.es \\ Tel: +34954486153 \\ Fax: +34954487248
}

\begin{abstract}
Nowadays, the building industry and its associated technologies are experiencing a period of rapid growth, which requires an equivalent growth regarding technologies in the field of vertical transportation. Therefore, the installation of synchronised elevator groups in modern buildings is a common practice in order to govern the dispatching, allocation and movement of the cars shaping the group. So, elevator control and management has become a major field of application for Artificial Intelligence approaches. Methodologies such as fuzzy logic, artificial neural networks, genetic algorithms, ant colonies, or multiagent systems are being successfully proposed in the scientific literature, and are being adopted by the leading elevator companies as elements that differentiate them from their competitors. In this sense, the most relevant companies are adopting strategies based on the protection of their discoveries and inventions as registered patents in different countries throughout the world. This paper presents a comprehensive state of the art of the most relevant recent patents on computer science applied to vertical transportation.
\end{abstract}

Keywords: Vertical transportation, elevator, lift, controller, artificial intelligence, fuzzy logic, neural network, genetic algorithm, double deck lift, hall call, traffic pattern, peak period

Short Running Title: vertical transportation in buildings 


\section{INTRODUCTION}

In recent years, the rapid growth of the building industry and associated technologies has demanded a similar growth in the field of vertical transportation. Nowadays the installation of synchronized elevator groups in modern buildings is common practice. Fig. (1) depicts an image of the Manhattan business centre.

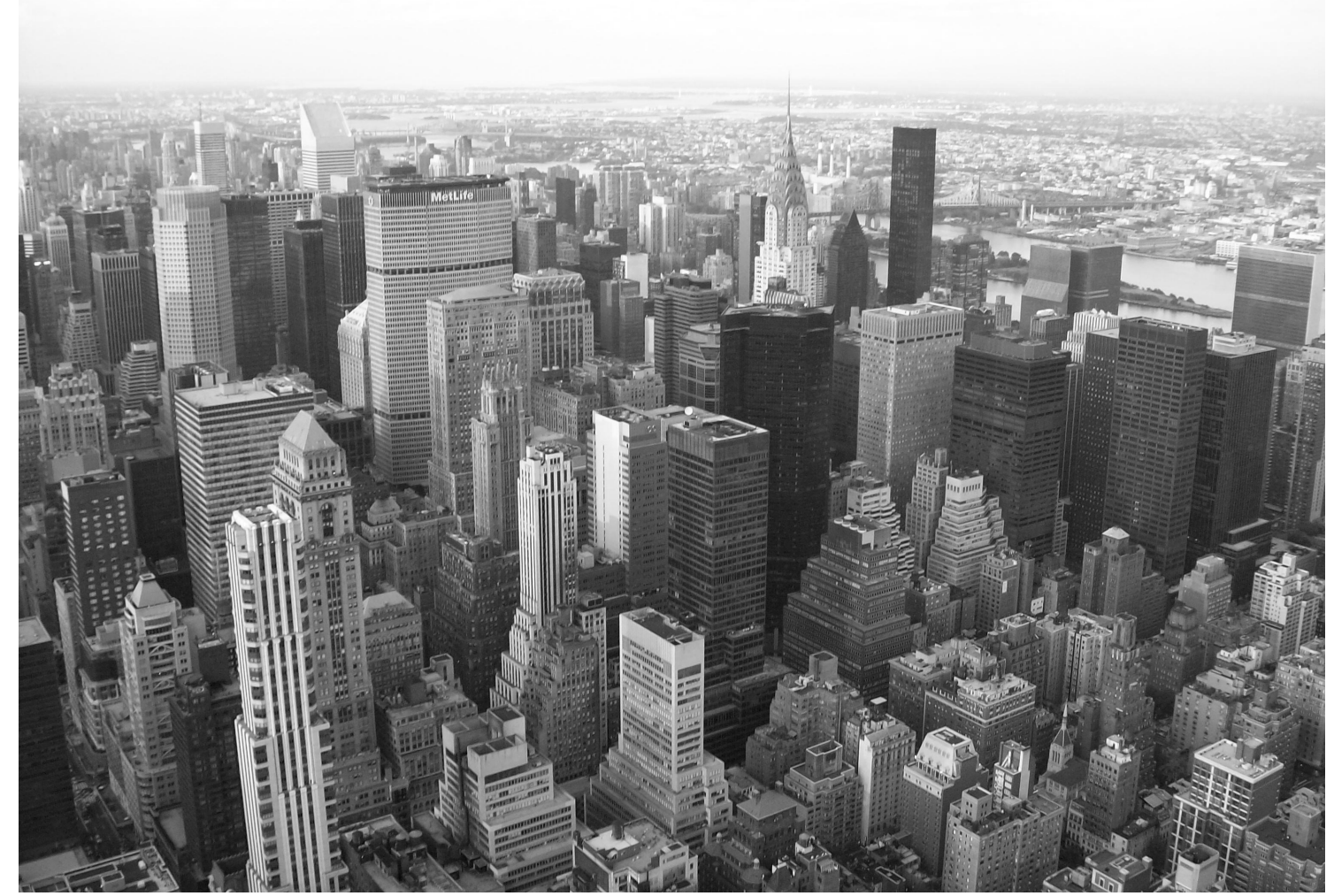

Fig. (1). Skyscrapers in the upper Manhattan business centre

The growing problem of vertical transportation has lead elevator engineers that work on vertical transportation to found professional associations such as the IAEE (International Association of Elevator Engineers) with a worldwide scope. Along the same lines, interest in the matter has moved the Chartered Institution of Building Services Engineers to publish a regular revision of the CIBSE Guide D focused on transportation systems in buildings [1].

However, the problem is constantly evolving and the challenges to be faced are growing. The management of complex groups of elevators serving many passengers who are wanting to travel to different floors at the same time, and sometimes sharing different buildings, requires the consideration of complex algorithms, control methods and advanced decision rules to be implemented in the controllers of the elevator group.

Furthermore, the numerous criteria to be considered make the problem particularly difficult. Traditionally, the main objective has been the minimisation of the passengers' waiting time to take a lift, with other significant performance criteria being the necessary limitation of maximum waiting times and the queue sizes of persons waiting for the lift. However, new efficiency criteria have recently arisen, such as the system's energy consumption. This criterion is supported by cost and sustainability factors. In fact, due to an increasingly environmentally aware society, a more rational consumption 
of energy will lead to a reduction of the costs of the electronic devices, as well as a reduced expenditure on energy.

Taking into account these considerations, elevator control and management has become a major field of application for Artificial Intelligence approaches. Methodologies such as fuzzy logic, artificial neural networks, genetic algorithms, ant colonies, or multiagent systems are being successfully proposed in the scientific literature, and are being adopted and "sold" by the leading elevator companies as elements that differentiate them from their competitors. Along these lines, the most relevant companies in the field are adopting strategies based on protecting their discoveries and inventions as registered patents in different countries such as the United States, the European Union, Canada, or Japan, amongst others. This is the case for the Otis Elevator Company, the Kone Corporation or Mitsubishi. This paper focuses on addressing and analysing such contributions, with the aim of generating a comprehensive state of the art on computer science patents applied to vertical transportation.

The rest of the paper follows with an overview of the type of vertical transportation problems that have arisen during recent years. The different problems are grouped into dedicated sections and discussed in detail. Each type of problem includes references to approaches in the scientific literature, as well as to the most relevant patents. The following sections thus include the most typical and traditional problems, consisting of allocating a landing call to a specific car of the elevator group; the problem of characterising the traffic in the building; the global control problem that tries to solve the two aforementioned issues at the same time; and other significant problems that are associated with special architectures, safety issues, or monitoring capabilities, amongst others.

\section{THE HALL CALL ALLOCATION PROBLEM}

This situation arises when a passenger wants to travel from one floor to another different floor in a building. The passenger makes a hall call to an elevator by pressing a landing call button installed at the floor and located near the cars of the elevator group.

After that, the elevator controller receives the call and identifies which one of the elevators in the elevator group is most suitable to serve the person that issued the call. This phenomenon is also known as "dispatching". The dispatcher's task is to monitor the hall and car calls and to control the movements of the elevators, to ensure that the passengers are collected promptly and transported rapidly to their destinations. The problem that must consequently be solved is how to select an elevator for each hall call that will minimise a preselected cost function. The dispatcher will usually aim to minimise the passenger waiting time, namely the mean time between the registration of the hall calls and the arrival of the car, but other performance measurements can also be considered, as has been already addressed in the introduction section.

Traditionally, the performance of a group of elevators is assessed analysing the passenger Average Waiting Time (AWT), the passenger Average Travel Time (ATT) and the passenger Average Journey Time (AJT), the latter being calculated as the sum of the former two. ATT is the time from when the responding elevator doors start to open until the time the doors start to open again at the passenger's destination. AWT is defined as the actual time a prospective passenger waits after registering a hall call (or 
entering the waiting queue if a call has already been registered) before the responding elevator doors start to open. Special consideration concerning maximum allowed delay must also be considered in order to provide a satisfactory service.

Energy criteria have recently taken on particular relevance, based on the increasing concern about sustainable development and the financial savings that can be produced in corporate buildings.

The problem can easily be proven NP-Hard independently of the criterion. So, most of the approaches are focused on artificial intelligence techniques. In fact, vertical transportation has become a major field of application for artificial intelligence approaches such as fuzzy logic, neural networks, genetic algorithms, and multiagent systems. All of which are techniques that are capable of providing better solutions than traditional controllers implementing dispatch expert rules that make use of simple IFELSE logical command sets.

\section{II.1. Approaches based on expert rules}

Traditionally, the main approach to manage a group of elevators has been using a set of predefined rules, which have been named "expert rules". Engineers with wide experience in vertical transportation proposed rules that aimed to govern the correct performance of the group. Undoubtedly, this situation became more complex as the complexity (and height) of the buildings grew. In fact, the problems of gaining expert knowledge, accounting for inconsistencies, and maintaining the underlying rule set, made the approach difficult to use. Furthermore, although certain traffic situations may look similar, they could require different dispatching decisions.

Most of the patents using expert rules are rather old and refer back to the 1980s and, in some cases, even the 1970s. It is however quite common to find new contributions from this field of research. Taking a look at the most recent contributions, we find among them a proposal by Kabushiki Kaisha Toshiba, presenting a controller based on a condition-instruction table which contains a set of predetermined control rules being defined by given conditions and given instructions, [2]. In accordance with a specific rule selected from the control rules, the elevator controller allows the detecting of a degree of establishment of the given conditions to provide a detected condition, and the generation, in accordance with the detected condition, of an elevator control instruction used for performing the group control.

A service/equipment equalization destination system for elevators has also been registered, [3]. The invention consists of an elevator car equalization computer program that includes software instructions enabling a computer to perform predetermined operations, a computer readable medium bearing the software instructions, and a computer system including a processor and a memory. The system receives inputs from the data input terminal containing information from the origin-destination travel. The assignment of an elevator to each location of the originating demand is carried out by forming temporary call response zones based upon the elevator assignments. The present system provides shorter average waiting times, transfer times, trip times, round trip times and equipment use, compared with traditional IF-ELSE rules. 
Inventio AG researchers proposed a master-slave system based on expert rules to determine the travel sequence planning for a group of elevators, [4]. The method determines the optimal travel sequence for an elevator installation that includes smart terminals, sending destination specific travel requests and other planning information to the job manager software for each elevator car. The job managers perform a situationbased search process to determine the optimal travel sequence plan for the associated elevator and then submit an offer to the terminal sending the travel request. The terminal compares offers and then books a selected one. The job managers respond to relevant changes in the situation upon which the plan is based, in order to determine an altered travel sequence and associated altered offer.

Researchers at Kone Corporation have presented an elevator control based on group size, [5]. The method allocates a car from the elevator group to a car call, based on a passenger data terminal, and an elevator group control system for controlling the elevators in response to signals from the passenger data terminal. This method requires the size and destination floor of the group of passengers to be input into the control system of the elevator group (from the historical data), allocating one or more elevators to the group of passengers by utilizing the data of the group's size and destination floor, and informing the members of the group of passengers about the allocation. The proposal's main idea is to make it possible for a passenger using an elevator to indicate that they belong to a group travelling at the same time from a landing level to one or more destination floors. Thus, according to the invention, when the passenger gives their destination floor, they can also inform the system that they belong to a group. This information is input using a passenger data terminal. The passenger data terminal may have a user interface with a menu or key for indicating that the passenger belongs to a group. It is also possible that only one member of the group inputs information into the passenger data terminal, telling the system how many members of the group are travelling to the destination floor. The destination floor elevator system, and the elevator group control system in particular, consequently receives information concerning how many passengers are travelling from the landing level to the destination floor. The elevator system can then send an elevator that has sufficient space for the entire group of passengers or, if necessary, several elevators for the whole group.

Very recently, researchers at Mitsubishi Company have proposed an elevator group controller based on expert rules, [6]. The proposal is related to the previous one, [5]. Here, an elevator group supervisory control system includes an estimation processing unit that determines an estimated in-cage load in departing from a departure floor, estimating at least one of the following measurements: speed, acceleration, and jerk rate of the car, in accordance with the estimated in-cage load, to determine an estimated arrival time. An assignment unit selects and assigns a car serving as a response to a hall call, on the basis of information from the estimation processing unit when the hall call is issued.

\section{II.2. Approaches based on fuzzy logic}

Fuzzy logic was first presented as a way of processing data by allowing partial set membership rather than crisp set membership or non-membership. Nowadays, fuzzy logic is widely used as a control methodology that incorporates a simple, rule-based "IF condition 1 AND condition 2 THEN consequence" approach to solving a control problem rather than attempting to model a system mathematically. Fuzzy logic is 
empirically-based, relying on manager/designer experience rather than their technical understanding of the system. Fuzzy logic was enthusiastically followed during the 1980 s and 1990 s by the vertical transportation industry. Since then, fuzzy logic has continued to be used. Most patents incorporate fuzzy techniques in an individual manner or as a part of more complex systems including hybrid methodologies. Fuzzy logic is considered as an Artificial Intelligence method providing a wider basis of analysis than traditional expert rules based on heuristics approaches.

Although fuzzy logic has mainly been used for global control strategies (Section 4), it has also been considered for the dispatching problem. For example, Otis researchers developed an elevator car assignment conditioned on minimum criteria based on fuzzy rules, [7]. An uncertainty filter was designed with input data, which is indicative of a fuzzy logic set having basis elements corresponding to cars of an elevator system having degrees of membership associated therewith corresponding to the utility of assigning the associated car to service a hall call. The uncertainty filter chooses a car corresponding to the term from the set having the highest degree of membership. The choice can be delayed until a predetermined amount of time has passed; or the degree of membership exceeds a predetermined amount; or the degree of certainty (the degree of membership of the chosen car divided by the sum of degrees of membership of the other cars) is more than a predetermined amount; or until either the degree of membership or the degree of certainty meets a value which varies as a function of time from when a hall call was registered.

The same company has also registered a fuzzy logic dispatcher that determines the elevator car assignment utility, [8]. In fact, the utility of assigning each car of an elevator system to service a hall call is determined by estimating the performance of each elevator car using a set of performance criteria. The performance criteria are then scaled by values that are indicative of the customer preferences for elevator system performance, in order to form scaled performance fuzzy sets. An assignment utility fuzzy set is formed from terms of the scaled performance fuzzy sets. The utility of assigning each car to service a particular hall call is then set equal to the maximum value of the degrees of membership of all of the terms of the scaled performance fuzzy sets. Alternatively, the utility of assigning each car to service a particular hall call can be set equal to the minimum value of the degrees of membership of all of the terms of the scaled performance fuzzy sets.

A particularly interesting situation emerges when the passenger destination is known by the system. This situation appears when the buttons in the car operating panel are the same inside and outside the car, resulting in the desired destination being indicated by the passenger service request prior to the passenger entering the elevator car.

In this sense, an elevator car dispatch including passenger destination information and a fuzzy logic algorithm has been registered [9]. The elevator system includes a controller that uses one of a set of fuzzy logic algorithms to assign an elevator car to service a passenger request. A disclosed example uses a passenger's desired destination as indicated by the passenger service request prior to the passenger entering the elevator car. The multiple fuzzy logic algorithms consist of a combination of relationships between an elevator car's current assignments, a passenger's desired destination, a source landing of the passenger's request, and a combination of them through a performance index. 
Also, researchers of Fujitec have patented a method for controlling a group of elevators using fuzzy rules, [10]. The system states the hall call allocation by applying fuzzy rule groups to the hall call, and selecting an optimum elevator car with a fuzzy inference applied, and assigning a call to the car. A set of fuzzy rule groups are successively applied according to respective priority orders previously given to the fuzzy rule groups. In such successive applications, a subsequent rule group is applied only when there is at least one car (excluding the car whose assignment aptitude is optimum) that has the difference in the assignment aptitude value for the current rule group, from that of an optimum car, no greater than a predetermined threshold value. This method includes an evaluation index calculation unit to carry out calculations of evaluation indexes, based on a traffic information signal, when a hall call occurs.

\section{II.3. Approaches based on neural networks}

Artificial neural networks are computational models based on a biological neural network analogy. They consist of an interconnected group of artificial neurons and they process information using a connectionist approach to computation. In most cases an artificial neural network is an adaptive system that changes its structure based on external or internal information that flows through the network during the apprenticeship phase. Artificial neural networks are useful to model complex relationships between inputs and outputs, or to find patterns in data, as well as to proceed with optimal decisions, as for example in the car dispatching problem that is being explored here.

Otis researchers presented an elevator control neural network system, [11], to state the car to be allocated to hall calls in a building served by a group. The system estimates the remaining response time for an elevator car to be assigned to a newly registered hall call. The neural network is standardised for use in any building, through the use of an upstream fixed length stop description that summarises the status of the building at the time of the new hall call registration, for one or more postulated paths of each and every car under consideration to answer the new hall call.

The same company later proposed a method for continuous learning by an artificial neural network used in the elevator dispatching system, [12]. The method can be viewed as the continuation of the company's previous work. In fact, the registered proposal consists of a method that trains a neural network used to estimate the remaining response time for the elevator to service a hall call. The training, which results in adjusting connection weights between nodes of the neural network, is performed while the elevator is in actual operation. The method is not restricted to any particular architecture of neural network. The method uses a cut-off to limit changes to the connection weights, and will scale the different inputs to the neural network, so that all inputs lie in a predetermined range. The method also provides for training in case the elevator is diverted from servicing the hall call by an intervening hall call.

\section{II.4. Approaches based on genetic algorithms}

Genetic algorithms are adaptive heuristic search algorithms premised on the evolutionary ideas of natural selection and genetics. The basic concept of genetic 
algorithms is designed to simulate processes in the natural system necessary for evolution, specifically those that follow the principles first laid down by Charles Darwin of survival of the fittest. As such they represent an intelligent exploitation of a random search within a defined search space to solve a problem. The genetic algorithm success in many fields of engineering has contributed to the popularity of this intelligent search process. The elevator industry has also greatly attracted interest in genetic algorithms. Kone Corporation has been researching the applicability of genetic algorithms to vertical transportation for many years. The report by the Helsinki University of Technology [13] addresses much of the research carried out. The paper shows an appropriate genetic algorithm based on a stochastic search method that aims to find near-optimal solutions. The method proposes the allocation of a landing call to a car forming a gene, and a combination of genes where each call is allocated to a car forming a chromosome. To start, random routes are generated, followed by a few of the best of the initial routes being selected. A new generation from the selected routes is created using inheritance, crossing over and mutation. Once again, the best routes are selected, and next generation is created. Finally, after some generations, the solution converges to the solution.

A genetic allocation method for allocating a landing call to one of several elevator cars in an elevator group is presented by researchers of the Kone Corporation [14], where cars move and stop within the area of several different floors.

The elevator travel routes are encoded into alternative chromosomes. Using genetic methods, alternative chromosomes are developed and the best chromosome is selected. Finally, the elevator group is controlled in accordance with the best chromosome. The algorithm makes use of building zoning: the floors served by the elevator group are divided into a first group and a second group. On the floors of the first group, landing calls are given as passenger-specific destination calls. On the floors of the second group, landing calls are given as floor-specific up/down calls. When the destination calls and up/down calls are encoded into the same chromosome, the best chromosome represents an allocation decision, in which the gene values indicate which elevator car is to serve each passenger and each up/down call.

Two similar patents were previously registered by some of the same inventors with the name "method for allocating landing calls", [15], and "method and apparatus for allocating passengers by a genetic algorithm", [16]. Analysing the latter [16], it seems a traditional genetic approach, whereas the former [15] presents certain novelty. The purpose of the invention is to achieve a new solution for allocating landing calls to the elevators in an elevator group, so that the control call received by each elevator changes as seldom as possible. This is achieved via genetic allocation, by using the allocation option selected in the preceding instance of allocation as one chromosome in the starting chromosome population in a new instance of allocation.

As we have previously stated, one of the current challenges is the optimisation of multicriteria functions, taking into consideration passenger waiting times as well as energy consumption. Kone corporation research recently began focusing on this line.

A genetic algorithm based on an elevator group control system that utilises a multiobjective criterion and that dynamically changes the process control environment has been presented, [17]. A bi-objective genetic elevator group control is presented, together 
with the performance results obtained from simulations concerning a high-rise office building. The results show that with this approach it is possible to regulate the service level of an elevator system, in terms of average passenger waiting time, so as to bring it up to a desired level while minimising energy consumption. The authors have also protected their invention as a patent, [18].

In addition to Kone Corporation research on genetic algorithms, other researchers have been carrying out research on genetic algorithms. A particularly interesting proposal presents a new concept for an elevator group control system that can change control settings according to individual floor utilization situations, [19]. The floor-attributebased control method carries out the assignment of a service car for a passenger. The assignment is done by: predicting waiting time or crowding in a car by using each car location, situation of other passengers, learned traffic demands of the building in the past, etc.; calculating evaluation values for each car from the predicted values; and selecting an optimum car by comparing the evaluation values. The optimisation of control parameters is carried out using an online genetic algorithm that tunes the parameters in order to minimise the waiting time, and seven control parameters related to multi-objective control were encoded into the chromosome. Population size (number of control parameter sets for a generation) was set to 30 . The simulator used lunchpeak traffic flow data that was measured in an actual building.

Researchers from LG Industrial Systems Company proposed an elevator group management system based on genetic algorithms, [20]. The authors claim that their proposal is capable of decreasing the average waiting time and the waiting generation probability by selecting more than two cars with high evaluation values, after evaluating each car using a synthetic evaluation function, and allocating the car proposed by the genetic algorithm. The genetic algorithm makes use of traditional crossover and mutation operators and an evaluation function for evaluating a gene of each solution. In order to appropriately include the estimate hall call generation probability for each car, a respectively different probability is applied to each different car. The estimate hall call generation probability is the probability that a hall call is generated within a time period.

Mitsubishi Company has also presented a group managing system for elevator cars based on genetic algorithms, [21]. The algorithm follows the traditional rules of genetic algorithms, including crossover and mutation operators. The best individuals are accumulated in a memory using additional registrations, in which the best individuals are additionally stored in the memory and deletions in which non-feasible solutions are deleted from the memory. The optimum set is selected from the accumulated sets, so that it can be efficiently found by the system.

\section{II.5. Approaches based on hybrid methods}

Hybrid methods are known for integrating several artificial intelligence techniques under the same application. They can combine genetic algorithms with fuzzy logic, neural networks with ant colony algorithms, as well as any other possible combination.

The scientific literature on vertical transportation has focused much attention on these types of methods. A good example is the proposal of an intelligent dispatching method based on ant colony algorithm and fuzzy neural network, [22]. The elevator group control system is based on a fuzzy neural network that adapts to various traffic flow 
modes. The proposal uses an ant colony algorithm to optimise the weights of the fuzzy neural network before training it with the back propagation algorithm. This approach can solve the problem of convergence of weights more effectively and avoids being trapped in local optimal values when trained just with the back propagation algorithm.

It is also easy to find good proposals in the patents field. Fujitec researchers proposed an elevator group supervisory control system based on a hybrid fuzzy-neural approach, [23]. The proposal includes an elevator group supervisory control system for selecting the most suitable car among a group of elevators. The algorithm comprises a temporary assigning means for temporarily assigning the car using a conventional method, such as a fuzzy group supervisory control based on group data representing the status of the elevator system at the moment when a new hall call is made; and a neural net for receiving numerical values converted from group data, including the judgement result of the temporary assigning means and outputting an assignment suitability of each elevator. By examining the output pattern of the neural network, the system decides which would be the most suitable elevator to assign to the hall call.

More recently, Kone Corporation has registered a method for controlling an elevator system based on the Enhanced Spacing Principle (which was first introduced by the same company), a genetic algorithm and statistical estimator of traffic, [24]. The invention produces a cost function for different route alternatives, in which the travelling time of each passenger is calculated. The calculation of the travelling time is performed taking into account the time spent while waiting for an elevator at a floor, the ride time in the elevator car, the delaying effect of active landing and car calls on the travelling time and the delaying effect of car calls given by new passengers entering at intermediate floors. The method brings advantages to the destination call system, but in the traditional up-down call system it is possible to utilise information provided by traffic statistics to predict the destination floor. Route alternatives are created using genetic algorithms. Once the route alternative giving the shortest average travelling time has been calculated, the elevators are controlled according to this route. In intensive traffic conditions, travelling time is optimised, whereas in quiet traffic conditions, passenger waiting time is optimised.

\section{II.6. Approaches based on other computer science techniques}

Other computer science approaches that cannot be classified as artificial intelligence techniques appear in the scientific literature (see references $[25,26]$ ), as well as in the vertical transportation patents databases. Some of them could also have been classified as expert rules but focus more on heuristic approaches. Due to specific differences compared with the aforementioned approaches, these other approaches have been dedicated a separate section.

This is the case of the group-control method for an elevator system with plural cages, [27], a patent registered by Hitachi. The proposal considers a multi-objective function that considers a performance index including the waiting time, the equal time-interval operation preferential zone, the riding time, the cage-load factor, the first-arriving cage preferential zone and the stop call, each of them weighted with specific parameters for the last five of the above-mentioned evaluation indexes that must be tuned. The generated hall call is allotted to a cage having a minimal evaluation value calculated by the aforesaid evaluation function. The control parameters are selected and set at 
appropriate values in accordance with the manner of use of a building installed with the elevator system.

Otis Elevator Company proposed an elevator sectoring for downpeak traffic conditions, [28]. As will be discussed in Section 3, during the down-peak time period people arrive at different halls on each floor with a probability that is associated to the population of the floor. Consequently, cars are assigned to sectors of an approximately equal number of floors, with the remaining floors being assigned to sectors near the lobby, with one car per sector. The proposal provides a dispatching strategy to serve elevator traffic in both the up and down directions, giving priority service to the down travelling traffic while providing limited service to the up travelling traffic. All floors requiring down service are given equal access to the system regardless of the floor's position within the building.

Kone Corporation has proposed a method for allocating landing calls in an elevator group, [29]. The proposal includes a set of decision rules together with a cost function to evaluate the suitability of the decision rule proposal. Specifically, the procedure considers an elevator bank that forms several allocation options. Each allocation option contains, for each active landing call, a call data item and an elevator data item, which together are used to determine which elevator should service the call. The value of a cost function is calculated for each allocation option; one or more of the allocation options is repeatedly changed regarding at least one data item, and the values of the cost functions of the new allocation options are calculated. Based on the values of the cost functions, the best allocation option is selected and the active elevator calls are allocated accordingly to the elevators in the elevator bank.

Mitsubishi has registered a method and elevator scheduler for the scheduling of a group of cars of an elevator system in buildings, [30]. The procedure schedules the cars of an elevator system, beginning the process whenever a newly arrived passenger presses an up or down button to generate a call for service. For each car, a first waiting time can be determined for all existing passengers if the car is assigned to service the call, based on the future status of the elevator system. Similarly, for each car a second waiting time can be determined for future passengers if the car is assigned to service the call, based on the landing pattern of the cars. Finally, for each car, the first and second waiting times are combined to produce an adjusted waiting time. The method is completed by assigning a particular car with the lowest adjusted waiting time to service the call, thus minimising the average waiting time for all passengers.

Otis Elevator Company researchers have proposed an approach for scheduling a group of elevators [31]. The proposal includes an information model that collects data such as passenger arrival time, arrival floor and departure floor, within a time window. A static problem is then solved for such a time window by minimising an objective function based on the total service time of all passengers in the period of analysis proposing the passenger-car assignments and car dispatching.

\section{II.7. Approaches based on other artificial intelligence techniques}

New approaches from artificial intelligence have recently arisen in the scientific literature. Some include considerable contributions to the car-call allocation problem, 
but we have not yet been able to find corresponding patents. They are multiagent system approaches, swarm particle algorithms, or immune systems algorithms, amongst others.

An interesting paper presents a distributed elevator group control system based on a multi-agent system that overcomes the computing bottleneck that exists in traditional collective elevator group control systems, [32]. The system is composed of C-agent, $\mathrm{HC}$-agent and $\mathrm{M}$-agent, which respectively correspond to elevator car, group controller, and a virtual management unit. The algorithm includes the architecture of a reticulate distributed system.

Another paper, [33] has proposed a particle swarm optimization (PSO) algorithm. The proposed algorithm includes a novel PSO-based dynamic scheduling algorithm that services zones that are determined by considering their expected round-trip time. The simulation results balance the round-trip time over the elevator system and further improves the service quality of the elevator system. Some of the same authors have also shown the efficiency of an artificial immune algorithm dispatcher when it is compared to the well-known THV-duplex algorithm, [34].

We have only shown a few examples from the scientific literature. New patents should therefore be expected over the coming years, contributing to the use of the most novel artificial intelligence techniques.

\section{TRAFFIC PATTERN IDENTIFICATION}

Most of the controllers are tuned depending on the type of traffic in the building at the time. Therefore, the elevator group control system needs to include a form of traffictype detector. This was traditionally done using a simple timer, but nowadays more sophisticated methods are being implemented.

Traffic patterns represent the mobility of a building population in its necessities of vertical transportation. Each building has a specific shape of its own traffic pattern. Following the indications of the CIBSE Guide [1], a typical traffic pattern can be depicted by Fig. (2), which shows the number of up landing calls and down calls registered during the working day in an office building. 


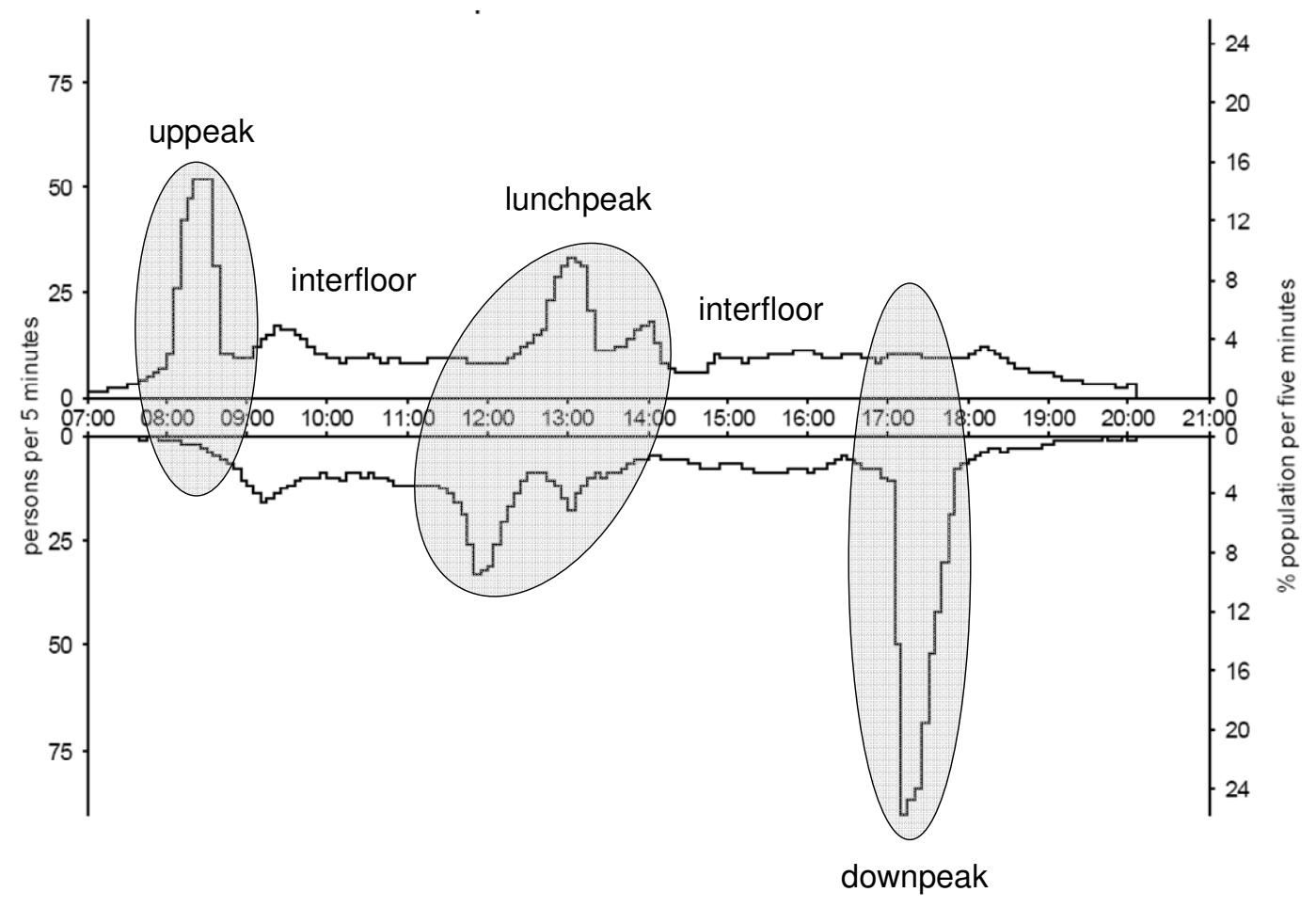

Fig. (2). Passenger demand rate for an office building

For a professional building, the traffic pattern will present a larger than average number of up landing calls at the start of the day. This is due to the building's workers arriving to start work, with the phase referred to as uppeak traffic. Late in the day there is the opposite phenomenon, and a larger than average number of down landing calls takes place. This corresponds to the building's population wanting to go home after a working day, and the traffic pattern is called downpeak.

In the middle of the day there are two joint phenomena, due to the appearance of up and down peaks. These depict a situation of people wanting to leave the building for lunch and people coming back after lunch. This period is called lunchpeak traffic.

Finally the rest of the day does not show any special tendency from any specific floor or from any specific stream. Generally, less traffic is registered too. This is called interfloor traffic.

The correct determination of the period of traffic pattern is a key factor, because most of the calculations used to estimate the performance indexes that were introduced in the previous sections depend on the correct identification of the corresponding peak period.

The traditional procedure for traffic pattern identification has been the utilisation of passenger traffic surveys. Human observers count passengers in and out of the lifts. This procedure has been implemented following two approaches. The first is called Survey from main terminal, when the observers count passengers in and out of the lifts at the main destination floor. This method is only suitable for uppeak identification. The alternative method is the In-car survey, when observers are situated in the lift car and count passengers in and out at every floor. 


\section{III.1. Approaches based on statistical techniques}

As can be easily deduced, the surveys' methods lead to inexact measurements and cannot be updated with the changes to building life. A more sophisticated alternative is the use of statistical techniques.

An elevator traffic flow prediction based on statistical learning theory is presented in [35]. The authors predict elevator traffic flow using least squares support vector machines, which is a type of support vector machine with quadratic loss function.

Kone Corporation has been researching several approaches based on calculus with statistical series for historical data. This is the case of the single exponential smoothing and the adaptive response rate single exponential smoothing (ARRSES) that was used by the Traffic Master System 9000, where the predictions were updated for each floor and journey direction, [36].

This company has also patented a methodology for the identification of incoming peak traffic for elevators, [37]. When the number of elevator passengers arriving at the lobby floor of the building exceeds a given peak traffic threshold value, the elevators serving the passengers are directed back to the lobby floors after the trip without a separate call. To allow for the faster detection of a peak traffic condition, use is made of information obtained from traditional peak hour identification as well as previously-recorded data obtained from statistics regarding the numbers of passengers.

Otis Elevator Company has also made use of statistical procedures to estimate flows of traffic, [38]. This is the case of an elevator traffic predictor that makes use of historical data checked for accurateness. The elevator system employs a microprocessor-based group controller that communicates with elevator cars in order to derive relative prediction values to be used in a scheme of final assignment. For example, cars are allocated to hall calls at different floors in the building, using appropriate dispatching strategies based on predicted traffic conditions. In the algorithm, the building population behaviour based on weekly events, daily events and real time events is predicted, with the events being used to derive relative prediction values based on the weighted summation of the real, daily and weekly values of the events. Weekly events are considered to be those that happened over a certain number of previous weeks (e.g. 10 weeks) on the same day of the week, while daily events are those that happened over the past few days (e.g. 5 days). Real time events are those which are more or less currently happening, with the time frame of reference being sufficiently short so that data can be produced that can effectively be considered "real time" data, typically covering only a matter of some minutes (e.g. 4 minutes) or even less. In determining which events recorded in the daily and weekly historic databases are to be used in deriving the predicted relative values, the degree of certainty of the data is evaluated using two threshold considerations, and factors based on previously recorded data that fails to have the requisite certainty are not used in the predictions.

Some years later the same inventor proposed new advances to his first algorithm for the detection of traffic behaviour. This is the case of the predictor elevator for traffic during peak conditions, [39], and his subsequent floor population detector for an elevator system, [40]. In the latter case, a computer controlled elevator system that includes signal-processing means dynamically computes each floor's population during the 
uppeak period by monitoring the boarding and deboarding counts and using those counts to update that floor's population figure throughout that period. After the period has been completed, the floor-by-floor information, which had been maintained in a table, is used to determine the final historic based floor population spread also using historic data based at least on the past several active days of population spread using exponential smoothing. As a form of verification, the lobby's figure, which typically should equal the total building population, is compared to the total of all of the upper floors' populations. The historically based derivation of the floor population is recorded and made available for use in other signal processing functions in the system, such as, for example, prediction methodology for dynamic channelling of the elevator cars, stored in a table for that current day's information.

However, pure statistical techniques are difficult to implement with an authentic real time scope. When a real time decision (that includes quick processing times) has to be taken, we need to resort to intelligent techniques.

\section{III.2. Approaches based on expert rules}

A previous step to artificial intelligence techniques is expert rules. Otis Elevator Company presented an "artificial intelligence" based learning system predicting "peakperiod" times for elevator dispatching, [41], which is more an expert rules system than an authentic artificial intelligence approach. The invention aims to determine the commencement and/or conclusion of uppeak and downpeak periods of operation. For example, for the commencement of the uppeak operation, a lobby boarding count is predicted, based on previously collected data concerning the number of passengers boarding the elevators at the lobby. The predicted lobby boarding count is compared with a predetermined threshold value. If the predicted lobby boarding count is greater than the predetermined threshold value, uppeak is commenced. In the preferred embodiment, the predetermined threshold value is a predetermined percentage of the building's population. Additionally, the present invention is aimed at a method of adjusting the threshold value based on current passenger traffic. For example, once uppeak is commenced, the load of the first few elevators leaving the lobby within a predetermined time interval is determined, and the threshold value is adjusted based on their determined load. If the determined load is greater than a certain percentage of the elevator car's capacity, indicative of starting uppeak too late, the threshold value is decreased. Similarly, if the determined load is less than a certain percentage of the elevator car's capacity, indicative of starting uppeak too soon, the threshold value is increased.

\section{III.3. Approaches based on fuzzy logic}

Fuzzy logic is a suitable technique to predict the behaviour of signals, meaning that its applicability to vertical transportation pattern detection is very relevant. In fact, most of the global control methods include fuzzy logic controllers to identify the current and incoming traffic. Here we address those references that consider fuzzy logic as a means of detecting traffic patterns without including the fuzzy logic detector as part of a global method.

Otis Elevator Company has proposed a fuzzy logic controller to determine the number of passengers entering and exiting an elevator car at a stop, [42]. The software forms 
three fuzzy logic sets representing temporary estimates of the number of entering passengers and forms three fuzzy logic sets indicative of temporary estimates of the number of exiting passengers. Finally, the sets are combined to form a single fuzzy logic set indicative of the number of entering passengers and a single fuzzy logic set indicative of the number of exiting passengers.

\section{GLOBAL CONTROL STRATEGIES}

We have nominated those methodologies that include a traffic predictor tool, together with a dispatcher system, global control strategies. The system is therefore capable of collecting information regarding the traffic in the building, and by using this information it proceeds with a car/hall call allocation procedure.

Most of the proposals include a fuzzy logic controller or a set of expert rules to identify the traffic pattern, which is combined with an artificial intelligence based method to propose the car/hall call allocation. The following subsections describe different approaches, according to methodology.

\section{IV.1. Approaches based on expert rules}

Expert rules start by carrying out an estimation of the traffic flow in the building, which leads to the identification of an algorithm selected from a set of alternatives, and consequently a specific car from the group is allocated to the registered hall call.

Otis Elevator Company proposed what was named an artificial intelligence based crowd sensing system for elevator car assignment, [43]. In reality it was a compendium of expert rules to control a group of elevators. The system employs a micro-processorbased group controller, which communicates with the cars of the system to determine the conditions of the cars, and which responds to hall calls registered at different landings in the building that are serviced by the cars under the control of the group controller, assigning hall calls to cars based on the summation for each car, relative to each call, a weighted summation of a plurality of system response factors, some indicative, and some not, of conditions of the car irrespective of the call being assigned, assigning varying "bonuses" and "penalties" to them in the weighted summation. Expert rules (based on [39]) are used to predict traffic levels and any crowd build-up at various floors, in order to assign more efficiently one or more cars to the floors where crowds are predicted, either parking them there, if they were empty or more appropriately assigning the car(s) to the hall calls. Traffic levels at various floors are predicted by collecting passenger numbers and car stop counts in real time and using real time and historic prediction for the traffic levels, with single exponential smoothing and/or linear exponential smoothing. Predicted passenger arrival counts are used to predict any crowd at fifteen-second intervals at floors where significant traffic is predicted. Crowd prediction is then adjusted for any hall call stops made and the number of passengers picked up by the cars. The crowd dynamics are matched to car assignment, with one or more cars being sent to crowded floor(s).

An interesting proposal was made by Inventio AG researchers, [44]. It consisted of a group control for elevators with load dependent control of the cars. The method begins when a call specifically assigned to a car that is unable to stop at the respective floor 
due to an expected overload, can be serviced subsequently by the same car. For this purpose first and second circuits, assigned to the floors, are provided. A selector scanning the floor and car call memories activates at every position the assigned first and second circuits, where the first circuit causes the car to pass the respective floor if an overload were to be generated at the stop. The second circuits assigned to the upward and downward directions are linked to each other in such a manner that, if an overload is established, the scanning by the selector of the floor and car call memories assigned in each case to the calls of opposite direction is prevented. After passing the nonserviceable floor and reaching the point of return of the direction of travel, the car travels without interruption back to the earlier disregarded floor, whereby the blocking of the scanning of the floor and car call memories of the calls in the opposite direction is cancelled.

\section{IV.2. Approaches based on fuzzy logic}

The scientific literature has always attracted interest in such approaches. A recent example of current interest is shown in [45], which presents a set of five dispatching algorithms in an integrated approach. The system is composed of several local control systems, which implement the dispatching algorithms and an elevator group control system (EGCS) based on fuzzy logic (FEGCS). The overall fuzzy implementation is composed of two subsystems, namely the traffic identifier and the dispatcher elevator subsystems. So, the FEGCS determines the best algorithm to be run under different traffic situations in each LCS, in order to reduce the passenger waiting time and the power consumption. So, this approach includes identification of the traffic and considers a multi-criteria objective, which is a very novel contribution to a global control problem. However the rules applied could be more precisely named as expert rules without a strict sense of optimality.

Patents regarding fuzzy logic approaches have been proposed by Mitsubishi, who have registered an elevator control system that uses a system of evaluation factors by fuzzy logic to govern the elevator group, [46]. The system includes a fuzzy rule base having fuzzy rules stored therein which govern the selection of an elevator cage to be assigned to respond to a call. A reasoning unit is provided for selecting the appropriate fuzzy rule to be applied to a cage. The reasoning unit selects the appropriate fuzzy rule according to evaluation factors such as the miss forecast rate and the estimation rate of the cages.

Otis Elevator Company proposed an elevator controller with an adaptive constraint generator based on fuzzy logic, [47]. The group controller includes an elevator dispatcher for controlling the operation of the elevator cars during single source traffic conditions, the elevator dispatcher having a constraint for limiting car assignments in response to the constraint; and an adaptive constraint generator for modifying a value of the constraint in response to an elevator control system output variable. In one embodiment, the group controller includes: a traffic and traffic rate estimator to provide fuzzy estimates of traffic and traffic rate; a fuzzy logic controller for providing a control parameter in response to the fuzzy estimates of traffic and traffic rate, the control parameter having a constraint for limiting a value of the control parameter; an adaptive constraint generator for modifying a value of the constraint in response to an elevator control system output variable; and an elevator dispatcher for controlling the operation of the elevator cars during single source traffic conditions in response to the control parameter. 
Later, the same company registered an open loop adaptive fuzzy logic controller for elevator dispatching, [48], where a group controller for controlling elevator cars in a building is composed of a traffic and traffic rate estimator that provides fuzzy estimations of traffic and traffic rate, together with an open loop fuzzy logic controller for providing a control parameter in response to the fuzzy estimations of traffic and traffic rate. The open loop fuzzy logic controller has membership functions for fuzzy sets of the control parameter, an adaptive controller for modifying the membership functions of the fuzzy sets of the control parameter in response to an elevator control system output variable, and an elevator dispatcher for controlling the operation of the elevator cars during single source traffic conditions in response to the control parameter.

LG Industrial Systems proposed a group management control method for elevator systems that employed traffic flow estimation by fuzzy logic using variable value preferences and decisional priorities, [49]. The system decides on a corresponding car to be allocated in accordance with a predetermined hall call based upon traffic flow and the fuzzy theory which are different from the characteristics of each building, which includes the steps of: a traffic flow collecting step for collecting information concerning a current traffic flow occurring at each hall call and car; a traffic flow study step for studying information collected at the traffic flow collecting step; a traffic flow anticipating step for anticipating a specific traffic flow after a predetermined time, based upon the information studied at the traffic flow study step; a specific mode judgement step for judging a specific mode corresponding to the traffic flow anticipated at the traffic anticipating step; an allocation control strategy establishment step for establishing control strategy in order to allocate a proper car based upon a specific mode judged at the specific mode judgement step and the information and role defined by the building manager; a comprehensive evaluation function operation step for operating a comprehensive evaluation function for each car when a hall call occurs; an allocation possible car selection step for selecting a predetermined car possible for a hall call based upon the comprehensive evaluation function operated at the comprehensive evaluation function operation step; a control item value operation step for operating an input value per control item for an allocated possible car selected at the allocation possible car selection step; and an allocated car decision step for deciding on a car based upon a predetermined estimated rule using an input value per control item operated at the control item value operation step.

\section{SPECIAL ARCHITECTURES: DOUBLE-DECK, MULTIPLE-DECK, AND MULTI-DOOR CARS}

This section refers to special architectures that often appear in vertical transportation. They all pursue the same objective: to gain space in the building structure, thus allowing an improvement in several performance measures such as handling capacity, round trip time, waiting times, energy consumption, etc.

\section{V.1. Constructive solutions: double-deck lifts}

The first of the special architectures to be analysed refers to double-deck lifts. This type of lift is unusual in Europe but very common in the USA on high-rise buildings. They 
comprise two passenger cars, one above the other, that are connected to a single suspension/drive system. This structure allows the car to serve two adjacent floors simultaneously [see Fig. (3) for an example]. The upper floor is served by the upper cab, while the lower floor is served by the lower cab. During peak periods the cab area is arranged to serve even and odd numbered floors respectively, although passengers must be guided adequately. The main advantage of double-deck lifts is that the shaft handling capacity is increased because there are two lifts in the same shaft. Special control systems must be designed for such a type of structure. The scientific literature has been attracting research on such types of architecture over recent years. An example is a group supervisory control system that uses genetic network programming, whose optimisation and performance evaluation are done through simulations, [50]. First of all, optimisation of the genetic network programming for the double-deck system is executed. Secondly, the performance of the proposed method is evaluated by comparing it with conventional methods, and the obtained control rules in genetic network programming are studied. Finally, the reduction of space requirements compared with single-deck elevator systems is confirmed.

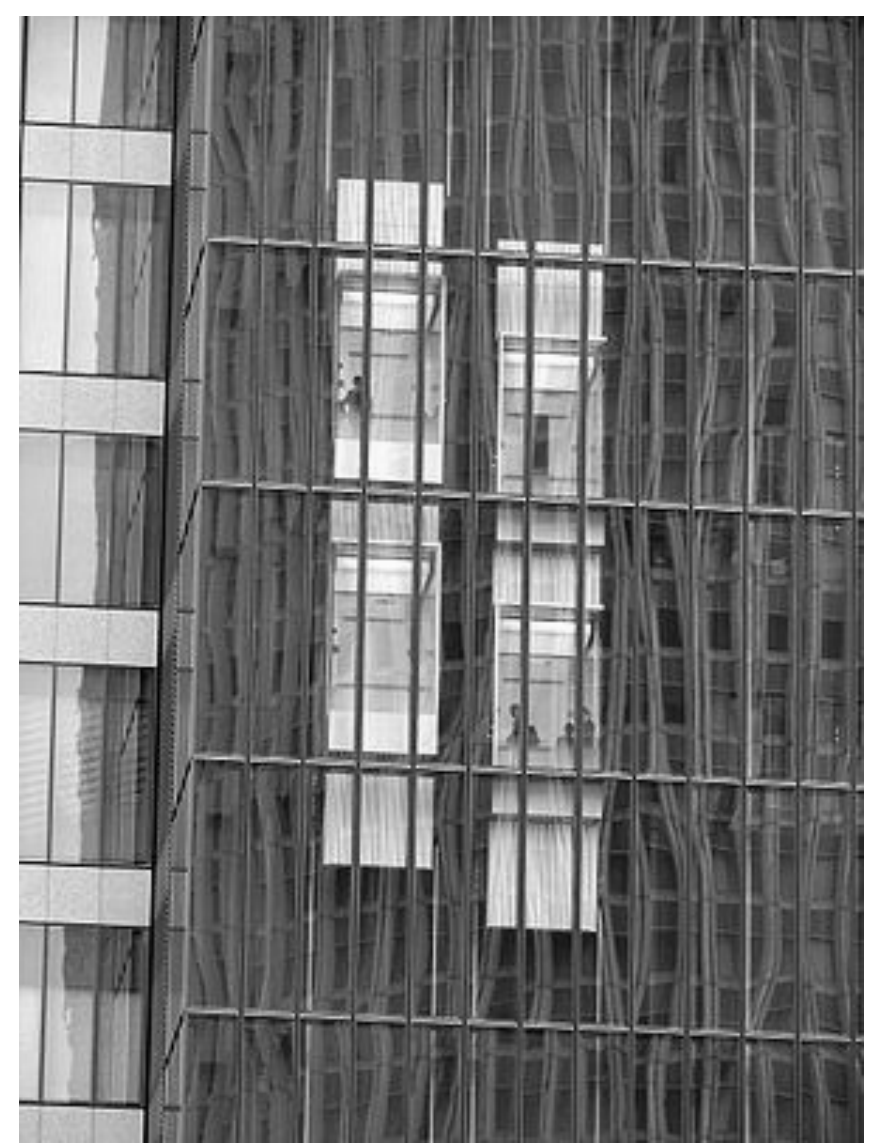

Fig. (3). A group of double-deck lifts at Midland Square, Nagoya, Japan

All the references that we have found in patent databases refer to the hall call allocation problem for this special type of elevator. This is the case of a double deck elevator allocation controlling system, [51], where a set of work rules are collected in an allocation control unit. When a predetermined specified floor is registered in an elevator hall call registering unit, each cage call registering unit is capable of registering a cage call to both the upper and lower cages in the double operation mode as far as the specified floor is concerned. The operation control unit in each elevator controls the operations of the upper and lower cages, and is able to respond to the elevator hall call 
to the specified floor, which is allocated by the allocation control unit, and to a cage call to the specified floor which is registered in the cage call registering unit.

Otis Elevator Company has shown an interest in such architecture by registering a control system and control method for the reassignment of the cars of a double-deck elevator, [52]. The control device includes a group control system that assigns the upper and lower decks to respond to car calls from the upper and lower decks and boarding hall calls from the different floors. The control device also includes an assignment control device that determines whether a set of the car calls and boarding hall calls can be responded to simultaneously and which directs the group control device to reassign the upper and lower decks to respond to the set of car calls and boarding hall calls simultaneously.

A method for controlling an elevator installation with multiple cars has also been registered, [53]. The proposal considers an elevator installation with multiple deck cars that serves several floors simultaneously with one stop, which is controlled in such a way that the travel requests are allocated to the most suitable elevator car of the elevator group, and the allocation of a travel request from a starting-point floor to a destination floor to a car deck of the elevator car takes place shortly before reaching the startingpoint floor. A travel request is also redistributed or allocated to another deck at any time up until shortly before it reaches the starting-point floor. The allocation of the travel request is carried out dependent on: general criteria; allocated travel requests for the region of the starting-point floor; and allocated travel requests for the region of the destination floor.

However, most of the research has been repeated by Kone Corporation. One of its first developments corresponds to a double-deck elevator group controller for call allocation based on monitored passenger flow and elevator status, [54]. The procedure for controlling the elevator group consists of allocating landing calls to elevators and elevator decks in such a way that passenger journey time is optimised. The time of the call and the estimated time of arrival at the destination floor are taken into account. Passenger flow and elevator status within the elevator group are monitored and the passenger wait time and arrival time are estimated. The best elevator is selected to minimise passenger wait and ride time. The best deck is then selected based on the estimated wait time and ride time, in order that the passenger journey time may be minimised.

The company's subsequent research has lead to the development of an advanced genetic procedure for multi-deck elevator call allocation, [55]. The genetic procedure considers chromosomes for the car allocations, each of which contains a car data item and an elevator direction data item for each active landing call, and these data (genes of the chromosome) together define a car to serve each landing call as well as a collective control direction for the elevator. For each chromosome, a fitness function value is determined. Following genetic rules, one or more of the chromosomes are selected and altered with respect to at least one gene, and fitness function values are determined for the new chromosomes. The process of altering the chromosomes, selecting chromosomes and determining fitness functions is continued until a termination criterion is met based on the fitness function values. Finally, the most suitable chromosome is selected and the calls are allocated to the elevators and cars in the elevator group, in accordance with the solution. 


\section{V.2. Constructive solutions: multi-door lifts}

Another possibility consists of lifts with more than one door. This constructive solution allows two twin buildings to be served at the same time. For example, one of them is served using the left door and the other one using the right door. Normally, access to these different buildings is protected with special keys or with identification codes. The solution can also include more than two doors providing service to more than two buildings. These are known as multi-door lifts. The multi-door option can be combined with double-deck lifts, thus significantly increasing the system's handling capacity.

We have found an interesting contribution from Kone Corporation, concerning the proposal of a method for the allocation of passengers in an elevator group considering several elevator lobbies and multi-door elevators, [56]. Each passenger gives their destination floor by means of a call input device, so that the passenger's starting and destination floors are thereby defined. The assignment procedure includes a genetic algorithm dispatcher, which also includes the possibility of double-deck lifts to gain shaft handling capacity.

\section{OTHER ISSUES: SAFETY SYSTEMS, MONITORING AND INTERFACING, AND PLANNING TOOLS}

\section{VI.1. Safety systems}

Elevators are systems that are subject to strong regulations that depend on the legal system of the corresponding country. However, constructors are permanently designing new safety elements that go beyond the regulations and that can be "sold" as an element of added value.

Kone Corporation has proposed a device to improve an elevator's safety system, [57]. The safety system of the invention comprises an electric safety device, which monitors the velocity and position of the elevator in the elevator shaft. The safety device consists of a computer that is able to stop the elevator, using the brake of the hoisting machine or an optional car brake. The basic idea of the invention's safety system is to form a continuous limit curve that controls elevator speed. The limit curve defines the limits of permitted elevator motion, which are determined on the basis of the nominal speed of the elevator and the location of the car. The safety system of the invention comprises measuring means, for the continuous measurement of elevator motion data and a safety device that receives data about the motion of the elevator, calculating its velocity at each instant of time by using the elevator motion data and watching the elevator motion to ensure that it remains within the allowed limit curve. Moreover, the safety system comprises a stopping device that will stop the uncontrolled motion of the car if the elevator motion exceeds the limit curve set for it.

Mitsubishi has registered an emergency stop system, [58], which is formed by: a status sensor for detecting the operation of a car; a brake device for braking the car; a brake controller for outputting a signal to operate the brake device based on a signal detected by the state sensor; and an uninterruptible power supply device for supplying electric power to the sensor, the brake device, and the controller. The controller has: a signal 
processing/calculating unit for calculating the deceleration of the car based on the signal detected by the sensor; a command value calculating unit for calculating a command value for operating the brake device based on the deceleration of the car calculated by the processing/ calculating unit; and a power monitoring device for monitoring the state of the uninterruptible power supply device.

A constructive solution to increase the shaft handling capacity is based on distributing two independent cars in a single shaft. However, this solution includes a potential risk that must be minimised. Mitsubishi proposed a system controller for two elevator cars in a single shaft, [59]. The proposal consists of a system controller for efficient group supervisory control while avoiding collisions between two elevator cars in service in a single elevator shaft. The elevator system controller includes a risk calculating unit which calculates the risk of a collision between elevator cars in the same shaft when the elevator cars are responding to a new call for service, a car assigning unit for assignment of an elevator car to respond to the new call based on the risk of collision, and an operation control unit for controlling operation of the elevator cars based on the assignment by the car assigning unit. The risk of collision is calculated for each car, and the risk is recalculated based on the possibility of the withdrawal of one of the elevator cars to a position in the shaft where no collision can occur, based on a predicted arrival time of a car at the floor that is requiring service. Those cars that have a high risk of collision when the remaining cars in the same shaft cannot be withdrawn to a safe spot in time are removed as candidates for assignment to respond to the new call. An evaluation is carried out using several evaluation indexes, in addition to the risk of collision, to decide which car is to be assigned to respond to the new call. If the determination of a traffic condition indicates low usage of the elevator cars, one car in each shaft is forwarded to a rest position and paused.

Another problem arising in the safety aspects of lifts is the door-open system of the car. A neural network detection of obstructions within, and movement towards, elevator doors has been registered by researchers of Otis Elevator Company, [60]. The system includes a camera, with suitable illumination that provides images to a processing card which converts the images to numerical vectors and applies them to a neural network which is capable of providing a door-open signal in response to something, either moving or still, being in the path of the doors, or anything moving in the landing adjacent to the elevator in a manner that indicates intent to enter the elevator. The dooropen signal is provided to the elevator door controller so that the doors become or remain open in response to anything moving towards the elevator or anything actually in the door pathway.

Finally, we address a proposal that concerns comfort more than safety, in the form of a control system for hydraulic elevators using fuzzy logic and speed control, [61]. The proposal includes: a flow control valve for controlling the amount of oil in a hydraulic jack; a sensor device for detecting at least one measurement of oil temperature and load pressure and producing the corresponding input data; a correcting rule storing device for storing correcting rules of control instruction values corresponding to input data from the sensor device; a fuzzy reasoning processor for calculating control instruction values as fuzzy values from the input data and the correcting rules; a speed pattern correcting circuit for correcting the control instruction values based on the fuzzy values calculated by the fuzzy reasoning processor; and a speed controlling device for supplying the corrected control instruction values to the valve. The objective is to improve the comfort 
experienced by passengers travelling in hydraulic elevators due to the jerk experienced when the car comes to a stop.

\section{VI.2. Monitoring and interfacing systems}

Nowadays, microprocessors provide an inexpensive means of monitoring vertical transportation systems, and an appropriate way to accumulate data that could be used in traffic detention or even dispatching strategies. In fact, lift monitoring systems enable building owners or managers to self-manage lift systems, thus optimising the safety, reliability, breakdowns, waiting times, and energy consumption, etc.

A comprehensive example of the wide range of applications associated to good monitoring systems is the Otis Elevator Company proposal based on a video aided system for elevator control, providing elevator dispatch and door control based on passenger data received from a video monitoring system, [62]. The video monitoring system includes a video processor that is connected to receive video input from at least one video camera. The video processor tracks objects that are located within the video camera's field of vision, and calculates passenger data parameters associated with each tracked object. The elevator controller provides elevator dispatch, door control, and security functions based in part on passenger data provided by the video processor. The security functions may also be based in part on data from the access control systems.

Toshiba Elevator Company has registered a functional variable remote monitoring system and remote monitoring method for elevators, [63]. The device collects and analyses data on the points that have been stated to be monitored without allowing a service person to visit the site dependant on the systems. The elevator is connected to a monitoring centre through a communication network. An agent device is installed on the elevator for each system, separate from the monitoring device of a control panel. The agent device selectively collects the data on measurement arbitrarily set by the instruction from the monitoring centre from the control panel, and transmits it to the monitoring centre. As can be seen, the system can therefore collect and analyse the data on the points to be carefully monitored without requiring a service person to visit the site.

A novel proposal consists on the replacement of video cameras by sensors. The area of an elevator is monitored in 3D by means of a three-dimensional sensor, which allows the groups of passengers moving in the building to be configured, [64]. The device utilizes a three-dimensional semiconductor sensor in order to detect three-dimensional image information. The sensor includes: a mounted light source so that the elevator area to be monitored is covered by the illuminated area of the light source; a sensor group that is mounted in such a manner that it receives reflected light; and a processing chip for converting the electrical signals into image information. In addition, the appliance includes a processing device that is connected to the semiconductor sensor in order to make the three-dimensional image information available. The processing device processes the image information in order to obtain status information that represents the status of the elevator area to be monitored.

A proposal to control door movement consists of a method for monitoring a manual elevator door system, proposed by S. Kamani and J. Sanches [65]. The contribution makes use of a set of sensor signals that are capable of detecting that an elevator car has 
stopped at a landing. The system determines whether the car door of the elevator car is open, and whether the hoistway door is unlocked or closed. It also determines whether a call has been assigned to the elevator car while the hoistway door was closed, providing a performance data signal in response to the collected information, where the performance data signal advises whether the manual elevator door system is in a nonalarm condition.

Finally, we address a two-part wireless communications system for elevator hallway fixtures [66] carried out by researchers from Otis Elevator Company. In their proposal, the elevator system hall fixtures such as lanterns, hall call button switches and lights, gongs, as well as the floor position indicators, are connected to a controller via wireless transceivers. A low power wireless system connects all fixtures on one hallway, with a higher power wireless system connecting each hallway with the appropriate controller. The controller also has a high power electromagnetic controller transceiver that is operatively associated with each of the floor transceivers, in order to exchange electromagnetic messages between each floor and the controller.

\section{VI.3. Elevator system planning tools}

Finally, in this section we address a simulating and planning tool that is used to select a system of elevators in a patent claimed by the University of Seville and MP Corporation, [67]. The tool consists of a system used to select a system of elevators to be installed in a building. The system comprises: means for obtaining data relating to the building; means for generating at least one user request model from the building data; means for obtaining a set of data for each elevator in the elevator system; control means which simulate the operation of the elevator system, according to the sets of data for the elevators in the elevator system and the generated user request model, and which determine the result of the simulation of the system; means for executing the aforementioned control means; and means for displaying the result of the simulation for each elevator system considered.

The tool allows the selection of the most suitable elevator group system configuration among a set of alternatives including mechanical characteristics, dimensions of the building and cars, number of cars in the group, and depending on the traffic pattern for each specific building.

\section{CURRENT AND FUTURE DEVELOPMENTS}

The vertical transportation problem refers to a person wanting to go from one floor to another floor within a building. However, the problem becomes increasingly more complex when the building is built higher and the population living (or working) in the building increases, thus making the exploration of advanced computer science techniques necessary. Nowadays, the progressive price increases of land in urban and business centres of the main cities in the world are leading to the construction of highrise buildings (commonly known as skyscrapers). In such buildings, the vertical transportation problem is extremely difficult to manage, particularly when everyone arrives at the same time at specific floors, wanting to travel to other floors. In order to solve such situations, the installation of specialised synchronized elevator groups in 
such buildings is common practice. Artificial Intelligence techniques are commonly used to overcome these difficulties. As has been shown in this survey, many artificial intelligence techniques have been successfully applied to deal with the problem, such as expert systems, fuzzy logic, artificial neural networks, genetic algorithms, ant colonies, particle swarm algorithms, immune system algorithms, or multiagent systems.

The majority of the main companies in the industry are starting to understand the importance of protecting their discoveries and inventions, and so they are registering their proposals in most of the countries where their commercial activity is relevant, such as the United States, the European Union, Canada, Japan, China, India, and other countries in the southeast of Asia and the Arabic peninsula. Otis Elevator Company, the Kone Corporation, Mitsubishi, Toshiba, Inventio AG, Macpuarsa Corporation (MP), and LG Industrial Systems Company, amongst others, have all done so. Most of the patents reviewed in this paper are (or were) included in the corresponding company catalogue.

The timeline patent production, as well as the different contributions concerning initial issues (heuristics, IF-ELSE rules, expert systems) and the most advanced Artificial Intelligence approaches (including genetic algorithms, fuzzy logic, or neural networks, amongst others), are addressing the ongoing research carried out in the industry. However, there is the need for new contributions, especially for those approaches addressed in section II.7 where we have been unable to find patents, such as multiagent systems, swarm particle algorithms, or immune systems algorithms amongst others. The contribution potential from multiagent systems emerges as an especially interesting field of work.

Table 1 summarises the different specific problems emerging in the vertical transportation generic problem and the approaches that are being used to tackle them. It can be viewed as a map representing the potential contribution from each specific field of knowledge as regarded by the industry. The references in the boxes represent the patents that have been registered to solve such a problem, using that specific computer science approach. 
Table 1. A classification of the most relevant computer science patents on vertical transportation

\begin{tabular}{|c|c|c|c|c|c|c|c|c|}
\hline & \multirow{2}{*}{$\begin{array}{c}\text { Hall call } \\
\text { allocation }\end{array}$} & \multirow{2}{*}{$\begin{array}{l}\text { Traffic } \\
\text { pattern }\end{array}$} & \multirow{2}{*}{$\begin{array}{l}\text { Global } \\
\text { control }\end{array}$} & \multirow{2}{*}{$\begin{array}{c}\text { Special } \\
\text { architect. }\end{array}$} & \multicolumn{3}{|c|}{ Other issues } & \multirow{2}{*}{ Total } \\
\hline & & & & & Safety & Monitoring & Planning & \\
\hline $\begin{array}{l}\text { Statistical } \\
\text { techniques }\end{array}$ & & $\begin{array}{l}{[37,38,} \\
39,40]\end{array}$ & & & & & & 4 \\
\hline Expert rules & $\begin{array}{l}{[2,3,4,5,} \\
6]\end{array}$ & [41] & {$[43,44]$} & [51] & & & & 9 \\
\hline Fuzzy logic & {$[7,8,9,10]$} & [42] & $\begin{array}{l}{[46,47,48,} \\
49]\end{array}$ & & {$[61]$} & & & 10 \\
\hline $\begin{array}{l}\text { Neural } \\
\text { networks }\end{array}$ & {$[11,12]$} & & & & {$[60]$} & & & 3 \\
\hline $\begin{array}{l}\text { Genetic } \\
\text { algorithms }\end{array}$ & $\begin{array}{l}{[14,15,16,} \\
18,19,20, \\
21]\end{array}$ & & & {$[55,56]$} & & & & 9 \\
\hline $\begin{array}{l}\text { Hybrid } \\
\text { methods }\end{array}$ & {$[23,24]$} & & & & & {$[63]$} & & 3 \\
\hline $\begin{array}{l}\text { Other comp. } \\
\text { sci. approach }\end{array}$ & $\begin{array}{l}{[27,28,29,} \\
30,31]\end{array}$ & & & $\begin{array}{l}{[52,53,} \\
54]\end{array}$ & $\begin{array}{l}{[57,58,} \\
59]\end{array}$ & $\begin{array}{l}{[62,64,65,} \\
66]\end{array}$ & {$[67]$} & 16 \\
\hline Total & 25 & 6 & 6 & 6 & 5 & 5 & 1 & 54 \\
\hline
\end{tabular}

Table 1 brings together the most relevant computer science patents on vertical transportation that have been presented and discussed in this survey. As can be seen from the table, most of the problems correspond to the hall call allocation problem when a car needs to be dispatched to serve a landing call (23 cases). The remaining problems also include a significant number of contributions, with a particular focus on the global control problem. Although a significant number of expert rules have been addressed, most of them correspond to old contributions (from the 1990s). Fuzzy logic and genetic algorithms (nine references each) are becoming the most commonly used artificial intelligence approaches for tackling the different situations currently emerging in vertical transportation problems. Furthermore, hybrid approaches usually include fuzzy rules as part of the hybrid method.

More and more research on such problems continues to be carried out, and future patents concerning the vertical transportation problem can be expected. Artificial intelligence in all its variants will continue to make important contributions to the field, providing the future with new developments and challenges. Undoubtedly, one of the developments must be to take on board the previous research and incorporate ambient intelligence in the vertical transport system. This ambient intelligence will lead to greater user-friendliness: more efficient services support, user-empowerment, and support for human interactions. The construction of a real ambient intelligence will require ubiquitous computing, ubiquitous communication and intelligent user interfaces, such as embedded algorithms for traffic pattern recognition or devices for vision or pattern recognition for lift group control and monitoring, among others. Following along this line, relevant topics that are to be incorporated in the near future (such as distributed artificial intelligence, vision or pattern recognition for lift group control and monitoring, human-machine interfaces, ubiquitous computing, ubiquitous communication, and embedded systems) should aim to attract the important research that is being carried out in the vertical transportation world.

\section{ACKNOWLEDGEMENTS}

The authors would like to thank MACPUAR, S.A. (Spanish elevator company) for the support of this research line since 2000. Also, the authors acknowledge the financial 
support given by the Andalusia Research Agency (Consejería de Innovación, Ciencia y Empresa, Junta de Andalucía) project ref. P07-TEP-02832, Spain.

\section{REFERENCES}

[1] The Chartered Institution of Building Services Engineers. Transportation systems in buildings CIBSE Guide D. Cibse Pub: London 2005.

[2] Yamaguchi, T.: US4760896 (1988).

[3] Newby, W.: US20056976560 (2005).

[4] Koehler, J., Schuster, K.: US20046793044 (2004).

[5] Ylinen, J., Kakko, M.: US20077281610 (2007).

[6] Hikita, S., Amano, M.: US2008431130 (2008).

[7] Sirag, D.J. Jr.: US5260526 (1993).

[8] Sirag, D.J. Jr.: US5248860 (1993).

[9] Stanley, J.A., Honma, H., Williams, D.S., Mori, T., Simcik, P.: US 20070045052 (A1) (2007).

[10] Sasaki, K., Yokota, K., Hattori, H., Sata, N.: US5022498 (1991).

[11] Whitehall, B.L., Sirag, Jr., D.J., Powell, B.A.: US5672853 (1997).

[12] Whitehall, B.L., Christy, T.M., Powell, B.A.: US5923004 (1999).

[13] Siikonen ML. On traffic planning methodology. Lift report. Helsinki University of Technology, Systems Analysis Laboratory 2001; 27 (3): 24-29.

[14] Tyni, T., Ylinen, J., Matela, M., Rintala, T.: US20067140472 (2006).

[15] Tyni, T., Ylinen, J.: US20046776264 (2004).

[16] Tyni, T., Ylinen, J.: US20056913117 (2005).

[17] Tyni, T., Ylinen, J. Evolutionary bi-objective optimisation in the elevator car routing problem. European Journal of Operational Research 2006; 169 (3): 960- 977.

[18] Tyni, T., Ylinen, J.: WO07099197 (2007).

[19] Fujino A, Tobita T, Segawa K, Yoneda K, Togawa A. An elevator group control system with floor-attribute control method and system optimization using genetic algorithms. IEEE Trans Industrial Elect 1997; 44(4): 546-552.

[20] Koh, E.L., Kim, J.O., Hahn, P.H.: US6000504 (1999).

[21] Tsuji, S.: US5780789 (1998).

[22] Liu J, Liu Y. Ant colony algorithm and fuzzy neural network-based intelligent dispatching algorithm of an elevator group control system. Proc 2007 IEEE International Conference on Control and Automation 2007; 2306-2310.

[23] Nakagawa, M., Sasaki, K., Markon, S., Nagashima, I., Midoritani, T., Tanabe, T., Ohta, N.: US5767461 (1998

[24] Siikonen, M.L., Ylinen, J.: WO05100223 (2005).

[25] Handi, M., Mulvaney, D.J. Prioritised $A^{*}$ search in real-time elevator dispatching. Control Engineering Practice 2007; 15 (2): 219-230.

[26] Luh PB, Xiong B, Chang SC. Group elevator scheduling with advance information for normal and emergency modes. IEEE Trans Automation Sci Eng 2008; 5(2): 245-258.

[27] Kuzunuki, S., Morita, Y., Yoneda, K., Ueshima, T., Tobita, T.: US4947965 (1990).

[28] Kameli, N., Collins, J.M.: US5480006 (1996).

[29] Tyni T, Ylinen J.: US5932852 (1999).

[30] Nikovski, D., Brand, M.: WO04113216 (2004).

[31] Atalla, M.J., Hsu, A.C., Luh, P.B., Luther, G.G., Xiong, B. WO/2006/113598 (A2) (2006).

[32] Wang ZT, Ji D-F, Qiao F, Wu Q-D. Modeling and scheduling elevator group control system based on MAS and negotiation mechanism of agents. Control and Decision 2007; 22(10): 1184-1188.

[33] Li Z, Tan HZ, Zhang Y. Particle swarm optimization applied to vertical traffic scheduling in buildings. Lecture Notes in Comp Sci 2007; 4692: 831-838.

[34] Li ZH, Tan HZ, Zhang YN, Mao ZY. Dynamic optimization of elevator group control based on artificial immune algorithm for inter-floor peak traffic during lunch-time. Control Theory Appl 2007; 24(2): 177-182. 
[35] Luo F, Xu YG, Cao JZ. Elevator traffic flow prediction with least squares supports vector machines. Fourth Int Conf on Machine Learning and Cybernetics, Guangzhou, China, August 18-21, 2005; 4266-4270.

[36] Siikonen ML. Elevator group control with artificial intelligence. Research reports A67 Helsinki University of Technology, Systems Analysis Laboratory, 1997.

[37] Tyni, T.: WO05000726 (2005).

[38] Kameli, N.: US5272288 (1993).

[39] Kameli, N.: US5276295 (1994)

[40] Kameli, N.: US5511635 (1996).

[41] Thangavelu, K.: US5035302 (1991).

[42] Sattar, S.A., Mizon, J.K., Sirag Jr D.J.: CA2085087 (1993).

[43] Thangavelu, K.: US5022497 (1991).

[44] Schroder, J.: US4878562 (1989).

[45] Muñoz DM, Llanos CH, Ayala-Rincón M, Van Els RH. Distributed approach to group control of elevator systems using fuzzy logic and FPGA implementation of dispatching algorithms. Eng Appl Artif Intel 2008; 21: 1309-1320.

[46] Amano, M.: US5233138 (1993).

[47] Thangavelu, K.: US5767460 (1998).

[48] Thangavelu, K.: WO1997019883 (1997).

[49] Kim, J.O.: US5679932 (1997).

[50] Hirasawa K, Eguchi T, Zhou J, Yu L, Hu J, Markon S. A double-deck elevator group supervisory control system using genetic network programming. IEEE Trans Syst, Man,Cybernet Part C: Applications and Reviews 2008; 38(4): 535-550.

[51] Ikeda, T., Yamaura, M., Sakakibara, K.: US20016176351 (2001).

[52] Mori, T., Bahjat, Z., Stanley, J., Ross, M., Sahara, M., Honma, H.: US20026419051 (2002).

[53] Kostka, M., Steinmann, K.: US20036508333 (2003).

[54] Siikonen, M.L.: US20026401874(2002)

[55] Ylinen, J., Tyni, T.: US20016293368 (2001).

[56] Matela, M.:US20056945365 (2005).

[57] Kattainen, A., Laasonen, T., Räsänen, M.: WO06082275 (2006).

[58] Kondo, R., Ueda, T., Kigawa, H., Okamoto, K.I., Yumura, T.: EP1958909 (A1) (2008)

[59] Hikita, S.: US20026364065 (2002).

[60] Cook, B.E., Pustelniak, R.D., Stagner, G.L.: US20077165655 (2007).

[61] Hatano, K.: US5266756 (1993).

[62] Lin, L., Xiong, Z., Finn, M.A., Peng, P.Y., Atalla, M., Kang, P., Misra, M., Netter, C.M.: GB2447829 (A) (2008).

[63] Yamaguchi, N., Suzuki, T.: JP2008081299 (A) (2008).

[64] Deplazes, R., Cortona, E.: US20060037818 (2006).

[65] Kamani, S., Sanches, J.: MX9707604 (A) (1998).

[66] Crenella, D., Gozzo, M.P., Grzybowski, R.R., Izard, J.M., Morgan, R.G., Slabinski, C.J. US6601679 (2003).

[67] Cortés, P., Onieva, L., Muñuzuri, J., Guadix, J., Fernández, P.: WO07088227 (2007). 